\title{
Head-Down Tilt Bed Rest Studies as a Terrestrial Analog for Spaceflight Associated Neuro-Ocular Syndrome
}

\begin{abstract}
Joshua Ong ${ }^{1}$, Andrew G. Lee ${ }^{2,3,4,5,6,7,7,9}$ and Heather E. Moss ${ }^{10,11 *}$
${ }^{1}$ University of Pittsburgh School of Medicine, Pittsburgh, PA, United States, ${ }^{2}$ Department of Ophthalmology, Houston Methodist Hospital, Houston, TX, United States, ${ }^{3}$ Baylor College of Medicine and the Center for Space Medicine, Houston, TX, United States, ${ }^{4}$ The Houston Methodist Research Institute, Houston Methodist Hospital, Houston, TX, United States, ${ }^{5}$ Departments of Ophthalmology, Neurology, and Neurosurgery, Weill Cornell Medicine, New York, NY, United States, ${ }^{6}$ Department of Ophthalmology, University of Texas Medical Branch, Galveston, TX, United States, ${ }^{7}$ University of Texas MD Anderson Cancer Center, Houston, TX, United States, ${ }^{8}$ Texas A and M College of Medicine, Bryan, TX, United States, ${ }^{9}$ Department of Ophthalmology, The University of lowa Hospitals and Clinics, lowa City, IA, United States, ${ }^{10}$ Departments of Ophthalmology, Stanford University, Palo Alto, CA, United States, "Departments of Neurology \& Neurosciences, Stanford University, Palo Alto, CA, United States
\end{abstract}

Astronauts who undergo prolonged periods of spaceflight may develop a unique constellation of neuro-ocular findings termed Spaceflight Associated Neuro-Ocular Syndrome (SANS). SANS is a disorder that is unique to spaceflight and has no terrestrial equivalent. The prevalence of SANS increases with increasing spaceflight duration and although there have been residual, structural, ocular changes noted, no irreversible or permanent visual loss has occurred after SANS, with the longest spaceflight to date being 14 months. These microgravity-induced findings are being actively investigated by the United States' National Aeronautics Space Administration (NASA) and SANS is a potential obstacle to future longer duration, manned, deep space flight missions. The pathophysiology of SANS remains incompletely understood but continues to be a subject of intense study by NASA and others. The study of SANS is of course partially limited by the small sample size of humans undergoing spaceflight. Therefore, identifying a terrestrial experimental model of SANS is imperative to facilitate its study and for testing of preventative measures and treatments. Head-down tilt bed rest (HDTBR) on Earth has emerged as one promising possibility. In this paper, we review the HDTBR as an analog for SANS pathogenesis; the clinical and imaging overlap between SANS and HDTBR studies; and potential SANS countermeasures that have been or could be tested with HDTBR.

Keywords: spaceflight associated neuro-ocular syndrome, head-down tilt bed rest, astronaut, space medicine, optic disc edema, microgravity, terrestrial analog, countermeasures

\section{INTRODUCTION}

During long-duration spaceflight (LDSF) missions, astronauts undergo a number of microgravity-induced physiological changes such as skeletal muscle atrophy, decreased bone mass, and height change (1-3). The neuro-ocular findings of LDSF include optic nerve head swelling, choroidal folds, cotton wool patches, a hyperopic shift, and retinal nerve fiber layer thickening (3). This constellation of findings was initially termed Visual Impairment and Intracranial Pressure (VIIP) syndrome based on the possibility of increased intracranial pressure (ICP) as the unifying 
mechanism. Over time however, the role of ICP alone in the condition has evolved and the term Spaceflight Associated Neuro-Ocular Syndrome (SANS) is likely more accurate (3).

SANS has an elevated "Likelihood and Consequence" rating from the NASA Human System Risk Board and accordingly will require mitigation for manned deep space journey and planetary missions of 1-3 years duration (4). In anticipation of these future LDSF exploration missions including flights to Mars, it is imperative to further study SANS, and develop strategies to mitigate it. One potential challenge to studying SANS is that it is uniquely associated with LDSF and the microgravity environment, and therefore is difficult to study on the scale required to understand the disease process. Thus, there is a need for terrestrial analogs (i.e., methods to induce SANS-like findings on earth) to accomplish this. Head-Down Tilt Bed Rest (HDTBR) is one such experimental model that has been used to simulate the effects of microgravity to study multiple physiological systems (5-14), and has become of interest as a terrestrial-based analog for studying SANS and testing potential preventative strategies and interventions, collectively referred to as countermeasures. SANS is characterized by an increased percentage of asymmetric or unilateral disc edema and does not have similar patient demographics found in terrestrial IIH $(3,15)$.

\section{CLINICAL PRESENTATION OF SANS}

Spaceflight is associated with reduced visual acuity in $\sim 29 \%$ of astronauts on short-duration ( $<6$ months) missions and $60 \%$ of astronauts after LDSF ( $\geq 6$ months) due to hyperopic shift $(4,16)$. In 2011, the first report of hyperopic shift, optic disc edema, cotton wool spots, choroidal folds, globe flattening, and retina nerve fiber layer thickening were reported in seven astronauts following a LDSF (6-month mission) on the International Space Station (ISS) (17). In-flight orbital ultrasound has revealed optic nerve sheath diameter dilation, and postflight lumbar punctures demonstrated slightly elevated but nearly normal opening cerebrospinal fluid (CSF) pressures in some individuals $(4,18)$. Intraocular pressure (IOP) increases initially during spaceflight, documented with a $20-25 \%$ increase $44 \mathrm{~min}$ into spaceflight (19). However, the Lifetime Surveillance of Astronaut Health gathered in-flight IOP data on 15 astronauts who underwent LDSF and found no significant changes between baseline pre-flight IOP, 30th day in-flight IOP, 30 days prior to returning to earth IOP, and post-flight IOP. Thus, suggesting that the acute elevation in IOP during immediate exposure to microgravity trends toward baseline shortly afterwards and stays at baseline throughout the mission (4). Prospective studies of SANS include the utilization of pre- and post-flight brain and orbital magnetic resonance imaging (MRI), retina and optic nerve optical coherence tomography (OCT), orbital ultrasound, cycloplegic refraction, funduscopic examination, and lumbar punctures (LP). Some of these measurements can be performed in-flight but some (e.g., LP, MRI) are logistically not feasible on the ISS (3).

Currently, astronauts utilize corrective plus sphere glasses (in the past "Space Anticipation Glasses"), onboard spaceflight missions as a countermeasure for the microgravity-induced hyperopic shifts $(4,20,21)$. Although no permanent vision loss has been reported, choroidal folds and posterior globe flattening may persist years after $\operatorname{LDSF}(3,17,22)$. Several astronauts have reported refractive changes that have yet to resolve (4).

\section{SANS PATHOPHYSIOLOGY HYPOTHESES}

During LDSF, astronauts are exposed to a myriad of factors that impose significant changes to the human mind and body. Microgravity, hypercarbia, and radiation must all be taken into account when understanding the physiologic impact of spaceflight. On Earth, there is a vertical hydrostatic pressure gradient from gravitational downward force resulting in different pressures throughout the body with relative increased pressure in the lower extremities that are closer to the Earth's center (23). A reduction in gravitational acceleration, such as that occurring with travel away from earth, reduces the hydrostatic pressure gradient and allows for a more uniform fluid redistribution in the body with a net shift of fluid toward the cephalad region $(3,23-$ 25) (Figure 1). It is an important distinction that astronauts onboard the ISS are still exposed to $\sim 90 \%$ of earth's ground gravitational pull (26). However, they are in a free fall state within the ISS as they orbit at tremendous velocities that counterbalance this gravitational pull, thus inducing the feeling of weightlessness and the physiological effects of microgravity including cephalad fluid migration $(3,23,25-30)$.

The exact pathophysiology of SANS continues to be an area of investigation, but multiple hypotheses have emerged. The first theory states that the ocular manifestations arise from high ICP resulting from venous hypertension due to cephalad fluid shifts that occur due to lack of gravity acting on the intravascular fluids. Post-flight MRIs of astronauts have shown findings similar to those seen in terrestrial high ICP states such as idiopathic intracranial hypertension (IIH) including posterior globe flattening and concavity of the pituitary dome (31). Jugular venous distension (JVD) occurs in microgravity studies supporting a hypothesis of venous congestion in the microgravity environment impeding cerebrospinal fluid absorption $(3,32,33)$. Furthermore, optic disc edema seen in SANS is a hallmark sign of terrestrial high $\operatorname{ICP}(3,34)$. Lastly, post-flight lumbar puncture readings in astronauts with optic disc edema showed an upper limit of high normal to slightly elevated post flight opening pressures $\left(21-28.5 \mathrm{~cm} \mathrm{H}_{2} 0\right)$, though these may not be representative as they were collected 12-57 days post flight (17). Interestingly, further studies of an astronaut with persistent asymmetric optic disc swelling for 180 days demonstrated only a mildly elevated opening pressure of $22 \mathrm{~cm} \mathrm{H}_{2} 06$ days postflight and normal pressure of $16 \mathrm{~cm} \mathrm{H}_{2} 0365$ days post-flight (35). In addition, terrestrial IIH symptoms typically include headache and pulsatile tinnitus, whereas the astronauts with optic disc edema do not report these symptoms (36). Interestingly, optic disc edema in astronauts may also persist up to 6 months after spaceflight, long after the proposed cephalic fluid shift resolves whereas the disc edema in IIH patients reduces relatively quickly after reducing pressure in the optic nerve sheath (e.g., Optic 


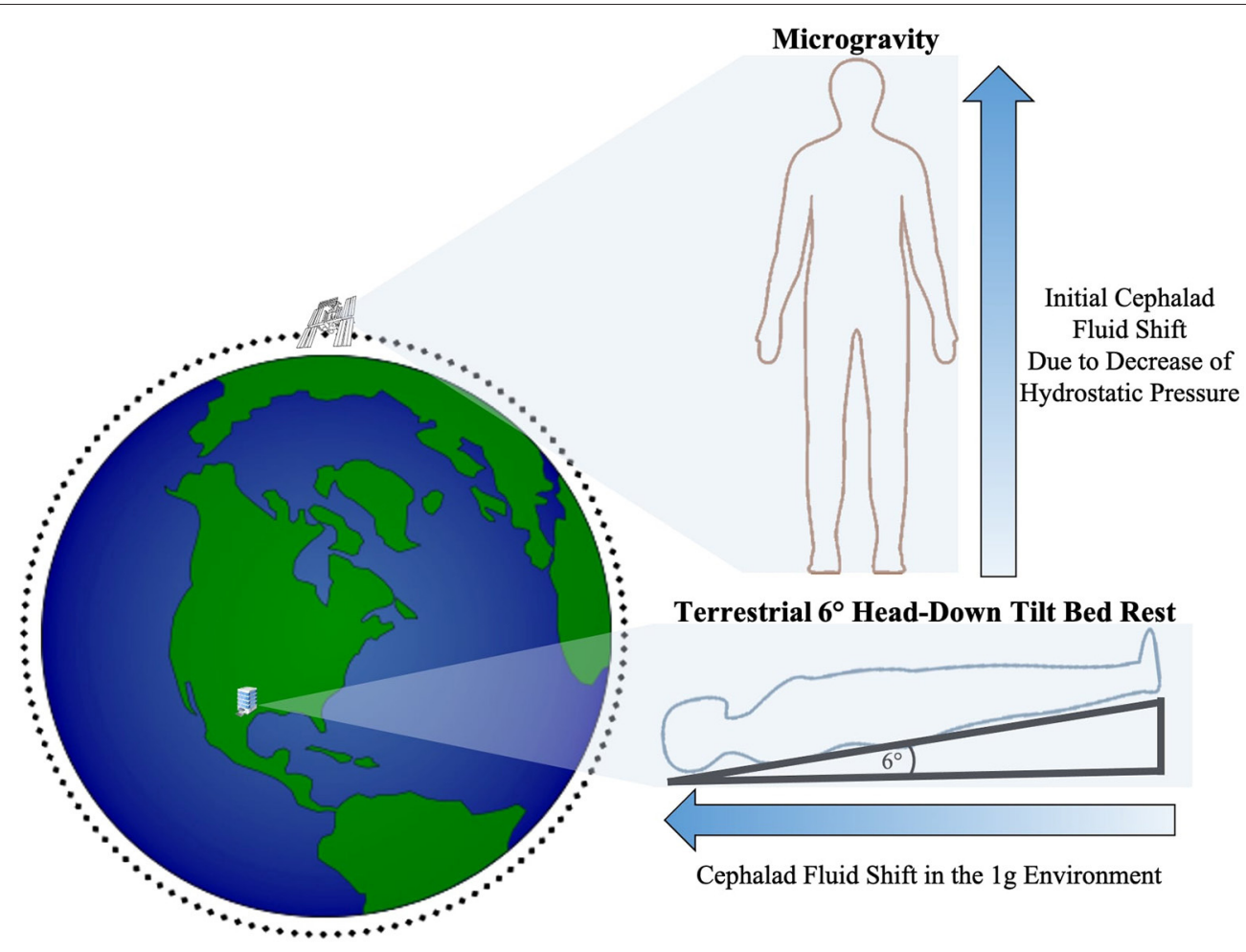

FIGURE 1 | Illustration of the initial cephalad fluid shift in the microgravity environment due to loss of hydrostatic pressure and cephalad fluid shift during terrestrial HDTBR studies (not drawn to scale).

nerve sheath fenestration) $(35,36)$. In addition, terrestrial IIH commonly presents with bilateral optic disc edema in women whereas SANS is characterized by an increased percentage of asymmetric or unilateral disc edema more common in men (3, $4,35,36)$. The collection of these clinical findings eventually led to the conclusion that venous hypertension leading to elevated ICP may not be the sole reason for SANS.

A second hypothesis postulates that in microgravity, CSF pressure increases locally within the optic nerve sheath due to a one-way valve-like mechanism allowing it to enter from the cranial subarachnoid space, but not exit. A similar mechanism of CSF accumulation has been theorized in the past with terrestrial IIH patients who present with persistent optic disc edema despite decreasing ICP (37). CSF biomarker studies have characterized a difference in the CSF composition between the subarachnoid spaces of the optic nerve and around the brain, thus furthering the notion that CSF equilibration between the spaces is incomplete (38). This theory of optic nerve sheath CSF compartmentalization may account for the persistence of optic disc edema in astronauts despite relatively normal to only slightly elevated ICP pressures. In addition, astronauts with SANS exhibit none of the typical terrestrial symptoms of increased ICP (e.g., headaches, diplopia, or tinnitus) $(17,36)$. Current study of the pathophysiology that induces the compartmentalization of CSF within the optic nerve sheath on Earth may give deeper insight into this theory for SANS (39).
A third hypothesis is that SANS is not due to elevated ICP but rather due to an upward shift of the brain during microgravity (40). Post-flight MRIs in astronauts have shown upward displacement of the optic chiasm (41). This observation is postulated to be due to slight rotation of the brain in microgravity, pulling the optic chiasm upwards and thereby exerting tension on the optic nerve. Because the optic nerve sheath (the dura that surrounds the optic nerve) is connected to the periosteum of the orbital bone, this exerts a compressive force on the optic nerve sheath, which in turn exerts a compressive force on the posterior aspect of the eye. Together these cause deformation of the eyeball (globe flattening) and expansion of the optic nerve sheath without ICP elevation (40). Supporting this hypothesis is a cohort study of twenty-two astronauts with post-flight MRI scans demonstrating a $0.80 \pm 0.74 \mathrm{~mm}$ (average $\pm \mathrm{SD}$ ) increase in optic nerve length from globe to chiasm compared to pre-flight scans. This was associated with forward displacement of the optic nerve head, which was related to duration of spaceflight and clinical signs of SANS (42).

While the pathogenesis behind SANS is not yet fully understood, the development of these hypotheses allows for proposal of potential experimental models of SANS on Earth (terrestrial analogs). When high ICP was thought to be the main cause, IIH was thought to be a close terrestrial analog but many authors believe that IIH is a flawed model for SANS. However, further characterization of SANS has now led to consideration 
TABLE 1 | Spaceflight associated neuro-ocular syndrome (SANS) findings demonstrated in head-down tilt bedrest studies.

\begin{tabular}{lccc}
\hline $\begin{array}{l}\text { Spaceflight associated } \\
\text { neuro-ocular syndrome } \\
\text { (SANS) findings }\end{array}$ & $\begin{array}{c}\text { Collective } \\
\text { head-down tilt } \\
\text { findings (up to } \\
\mathbf{7 0} \text { days) }\end{array}$ & $\begin{array}{c}\text { Parameters } \\
\text { (angle and } \\
\text { duration) }\end{array}$ & References \\
\hline Optic Nerve Sheath Distension & + & $6^{\circ}, 60$ min & $(48)$ \\
Retinal Nerve Layer Thickening & + & $6^{\circ}, 14,30, \& 70$ & $(49,50)$ \\
Optic Disc Edema & + & $6^{\circ}, 30$ days & $(50,51)$ \\
Choroidal Thickness Increase & + & $6^{\circ}, 60$ min & $(48,50)$ \\
(Possible) ICP Increase & + & $10^{\circ} \& 20^{\circ}, 5 \mathrm{~min}$ & $(52)$ \\
Choroidal Folds & - & - & - \\
Hyperopic Shift & - & - & - \\
Cotton Wool Spots & - & - & -
\end{tabular}

of other terrestrial analogs that may aid in understanding this neuro-ophthalmic phenomenon (3).

\section{HEAD-DOWN TILT BED REST STUDIES AND CLINICAL FINDINGS}

Terrestrial-based analogs for LDSF have been of longstanding interest in space medicine research as simulated microgravity is an efficient platform to observe physiology in extreme situations. Analogs such as supine bed rest, head-down tilt bed rest (HDTBR), wet immersion, dry immersion, and lower-extremity limb suspension have been previously applied as earthbound experimental models to further understand the human body during spaceflight (24).

HDTBR studies serve as a terrestrial analog by altering the vector of gravitational force on the body to generate a cephalad fluid shift similar to that seen in microgravity (43-45). Subjects lay supine on a bed that has been tilted to lower the head at a specific angle with the international standard angle established at $6^{\circ}(46)$. Though other techniques such as parabolic flight more closely simulate the actual microgravity condition, the brief duration of exposure is not sufficient for manifestation of SANSlike features. In contrast, long-duration HDTBR is feasible with study durations of 370 days having been successfully completed $(24,47)$. Furthermore, many outcomes analogous to those used by NASA for study of astronauts (MRI, OCT, LP etc.) can be measured in the HDT condition (Table 1).

One $6^{\circ}$ HDTBR study (14- \& 70-days) observed a mild increase in IOP with +1.42 and $+1.79 \mathrm{mmHg}$ from baseline, respectively. This study also saw an increase in OCT peripapillary retinal nerve thickening in the 70-day HDTBR compared to the 14 -day $(+11.50$ vs. $+4.69 \mu \mathrm{m}$, superior peripapillary retinal thickness), though overt optic disc edema was not apparent on clinic exam (49). It was postulated that this RNFL thickening represented early congestion of the optic nerve. Based on the observation that subjects with Ommaya reservoirs, an intraventricular catheter device that acts as a CSF conduit from the ventricle to the scalp, had a reduction of ICP from $14 \pm 2$ to $10 \pm 2 \mathrm{mmHg}$ when moving from a supine position without pillow to with pillow, the research team hypothesized that use of pillows and lifting the torso to eat meals may impact the cephalad venous congestion induced by HDTBR and thus the HDTBR protocol was modified with strict requirements prohibiting any lifting of the head and torso, including raising elbows for support to eat meals, or use of a standard pillow. The protocol was also modified to more closely mimic ISS conditions with a mild hypercapnic environment $\left(\mathrm{PCO}_{2} 3.8 \mathrm{mmHg}, 0.5 \%\right)$ compared to earth's surface $\left(\mathrm{PCO}_{2} 0.6 \mathrm{mmHg}, 0.04 \%\right)$, which was verified to not impact arterial $\mathrm{PCO}_{2}$ levels $(51,53)$. A $6^{\circ}, 30$-day HDTBR study using this modified protocol was associated with Frisén grade $1-2$ optic disc edema observed in $\sim 45 \%$ of subjects (51). This provided strong support for strict HDTBR studies in a hypercapnic environment as an experimental model to study the pathophysiology and test countermeasures for SANS. The same research group also compared healthy subjects undergoing strict HDTBR with 20 astronauts during 30 days in spaceflight (50). In this study they found a larger increase in peripapillary total retinal thickness in the strict HDTBR subjects than in the 20 astronauts with the average difference being $37 \mu \mathrm{m}$. Interestingly, choroid thickness showed the opposite pattern; there was a larger increase in astronauts compared to the strict HDTBR subjects with the average difference being $27 \mu \mathrm{m}$. Another study furthered this investigation of the mild hypercapnic environment with HDBTR and observed that individuals who developed SANS features from HDTBR demonstrated elevated reliance on visuals cues when tested on cognitive performance compared to non-SANS HDTBR individuals (54). The study raised concerns that SANS in astronauts may influence inflight performance for certain tasks or that SANS may be associated with cognitive changes. However, a recent strict HDTBR study in the mild hypercapnic environment of approximately $4 \mathrm{mmHg}$ $\mathrm{PCO}_{2}$ found no significant change in cerebrovascular reactivity, hypercapnic ventilatory response, or arterialized $\mathrm{PCO}_{2}$ (55). Interestingly, observations of cerebral perfusion throughout HDTBR demonstrated decrease in perfusion in all subjects with higher perfusion in the subjects that developed SANS symptoms compared to those who did not (56). With these recent findings, the role of the hypercapnic environment in SANS development remains an area for further study.

HDTBR studies have also been associated with short-term ocular findings that may have implications for astronaut neuroophthalmic health. A $7^{\circ}, 2$ min head-down tilt was associated with a decrease in choroidal pulsatile ocular blood flow, suggesting retinal hypoperfusion (57). A $6^{\circ}$, 12-h study HDTBR observed no significant global minimum rim width thinning compared to significant thinning seen in the seated position for $12 \mathrm{~h}$, although it was noted that Bruch's membrane opening height moved anteriorly during HDTBR. The study suggested that this attenuation in neuroretinal rim thinning in postural differences from seated to HDTBR may be due to translaminar pressure difference, which has been proposed as a contributing factor in glaucoma (58). A postural study on ambulatory neurosurgical patients with continuous ICP monitoring observed an increase in ICP during postural change from standing or supine to $10^{\circ} \& 20^{\circ}$ head-down tilt $(4,59) .6^{\circ}, 60$-min head-down 
TABLE 2 | Intraocular pressure findings during spaceflight and head-down tilt bedrest studies.

\begin{tabular}{llll}
\hline $\begin{array}{l}\text { IOP during } \\
\text { spaceflight } \\
\text { findings }\end{array}$ & $\begin{array}{l}\text { Head-down tilt } \\
\text { findings }\end{array}$ & $\begin{array}{l}\text { Parameters } \\
\text { (angle and } \\
\text { duration) }\end{array}$ & References \\
\hline $\begin{array}{l}\text { Acute IOP Increase } \\
\begin{array}{l}\text { Subsequent IOP } \\
\text { normalization to }\end{array}\end{array}$ & $\begin{array}{l}\text { Continued IOP } \\
\text { increase from } \\
\text { baseline }\end{array}$ & $6^{\circ}, 14 \& 70$ days & $(49)$ \\
\hline
\end{tabular}

tilt was associated with optic nerve sheath distension on orbital ultrasound, similar to that seen in astronauts after 1 month in-flight $(4,18,48)$, as well as increased subfoveal choroidal thickness on OCT (48). Certain SANS findings such as refractive shift, cotton wool spots, and choroidal folds have not yet been observed in individuals in HDTBR studies.

Some studies have identified possible risk factors for SANS. $15^{\circ}$, 21-min HDT was associated with higher peak IOP in moderate myopes $(19.8 \mathrm{mmHg}$ ) compared to emmetropes and low myopes (18.6 \& $18.7 \mathrm{mmHg}$, respectively) (60) (Table 2). In another study, single-nucleotide polymorphisms involved in vitamin $\mathrm{B}_{9}$ and $\mathrm{B}_{12}$ metabolism (5-methyltetrahydrofolatehomocysteine methyltransferase reductase (MTRR) $66 \mathrm{G}$ and serine hydroxymethyltransferase 1 (SHMT1) 1420C alleles) were associated with the magnitude of optic disc edema during strict HDTBR (61). These studies highlight the potential utility of HDTBR to generate hypotheses regarding SANS etiology, identify screening tests, as well as a possible role to empirically screen astronaut candidates.

\section{COUNTERMEASURE TESTING AND MONITORING DEVELOPMENT IN HEAD-DOWN TILT BED REST}

While advancing our understanding of SANS is an important application of HDTBR, the study model is also useful for testing possible countermeasures which may mitigate SANS. Lower body negative pressure (LBNP) device is a non-invasive technique that surrounds the pelvic area and legs and simulates the effects of gravity by inducing fluid redistribution from the cephalad region toward the lower body (52). LBNP has been studied during spaceflight as a countermeasure for cardiovascular responses toward microgravity-induced fluid shifts and orthostatic intolerance upon returning to earth's gravity (62). LBNP of $-20 \mathrm{mmHg}$ during a 5 -h HDTBR was associated with less increase in optic nerve sheath diameter as well as orbital and intracranial cerebrospinal fluid volume measured with MRI compared to the control group (63). Another HDTBR countermeasure study found a $40 \%$ decrease in increased choroid volume in association with use of $-20 \mathrm{mmHg}$ LBNP for 8 h/day during 3 days of strict $6^{\circ}$ HDTBR. Although the choroid volume was still increased during this HDTBR study, this significant attenuation suggests that LBNP may be an effective countermeasure for SANS (64). Thigh cuffs are also non-invasive devices that have been tested during spaceflight (65). Deflated cuffs are typically placed around the thigh and inflated at specific pressures (often ranging from 40 to $60 \mathrm{mmHg}$ ) to limit the amount of fluid flow to the upper body (65). Interestingly, a $15^{\circ}$ head-down tilt study utilizing bilateral thigh cuffs at $60 \mathrm{mmHg}$ for $10 \mathrm{~min}$ was not associated with significant differences in peripapillary choroidal thickness or optic nerve sheath diameter between cuffed individuals and controls (66).

To mitigate the atrophying effect of microgravity on skeletal muscle, astronauts undergo $2.5 \mathrm{~h}$ of intensive resistance and aerobic exercise nearly every day onboard the ISS (67). However, implementation of NASA's integrated resistance and aerobic training (iRAT) protocol during 70-day non-hypercarbic strict $6^{\circ}$ HDTBR was not associated with a significant difference in retinal thickening or signs of optic disc edema compared to a control HDTBR group who did not exercise, though IOP was slightly higher $(<1 \mathrm{~mm} \mathrm{Hg})$ in the exercise group (68). Interestingly, $15^{\circ}$ head down tilt for less than an hour was associated with a decrease in IOP in subjects undergoing either moderate-intensity aerobic, resistance, or high-intensity interval aerobic exercise (69). These differences highlight how the impacts of countermeasures are affected by the duration and angle of HDTBR. Integration of results from different HDTBR models is likely necessary to understand the short- and long-term effects.

HDTBR has been leveraged to develop devices for monitoring SANS features that are feasible for inflight use. For example, monitoring ICP during spaceflight is a topic of interest, with invasive measurements such as lumbar puncture currently not feasible inflight. Therefore, deployment of non-invasive devices to measure ICP is desirable $(70,71)$. Otoacoustic Emission (OAE) phase change is a candidate non-invasive method to monitor ICP. Changes to the positioning and tension of the middle ear caused by ICP changes are detected by a OAE probe placed at the opening of the ear canal. The OAE technique has been tested in head-down tilt and helps guide interpretation of OAE measurements taken aboard the ISS (72). Ocular vestibular evoked myogenic potentials (oVEMPs), which record extraocular muscle activity during vestibular stimulation, were observed to be associated with head-down tilt magnitude, supporting oVEMPs as a non-invasive ICP monitoring tool (73).

For years during spaceflight, OCT has been crucial in quantifying the retinal nerve fiber layer (RNFL) changes to supplement the clinical observations of SANS. In December 2018, OCT angiography (OCTA) became available on the ISS (3). OCTA is a non-invasive, high resolution ocular imaging technique that measures blood flow information and provides angiographic data that corresponds to retinal and choroidal vessels (74). Compared to invasive, contrast enhanced, fundus fluorescein angiography and indocyanine green angiography, OCTA is a non-invasive approach to blood flow visualization in the retina and choroid and may play an increasingly important role in evaluating the ocular vasculature in SANS $(3,75)$. This technology onboard the ISS will offer a clearer understanding of the volumetric shifts during spaceflight, particularly in the setting of the SANS cephalad fluid theory. Utilizing OCTA with HDTBR studies and comparing these results to ISS OCTA will provide 
novel information on HDTBR's ability to mimic spaceflightinduced fluid shifts within the retina vasculature.

\section{LIMITATIONS OF HDTBR}

Although HDTBR is a promising terrestrial analog for investigating SANS and for testing the efficacy of any potential countermeasures, certain limitations must be taken into account. One notable limitation is the small sample size of HDTBR studies, reflecting their time and resource intensive nature. The commitment of subjects to participate for months while maintaining a strict position that may be uncomfortable and induce headaches, which may make recruitment challenging (76). Generalizing HDTBR observations to LDSF and SANS may be limited by differences between terrestrial subjects and astronauts. Two years before a spaceflight mission, astronauts undergo intensive pre-flight strength and aerobic conditioning (77). Matching HDTBR subjects to a similar training regiments prior to HDTBR would be ideal but impractical. Screening protocols, such as a modified Air Force Class III physical exam and clearance by NASA Test Subject Screening facility have been applied to address this and select subjects similar to astronauts in terms of age, height, weight and physical fitness (48).

The variability in conditions for various studies also makes it challenging to integrate the findings of HDTBR with SANS. While a uniform, international standard $6^{\circ}$ angle has been established, the strictness of HDT positioning is not standardized and reports of studies often do not include sufficient detail to permit replication. There are conditions induced by HDTBR that are inconsistent with spaceflight, such as subjects having their posterior side in contact with the bed at all times. It is not known how these features impact results and the homology between HDTBR and SANS.

\section{DISCUSSION AND FUTURE DIRECTIONS}

A planned manned mission to Mars will require even longer duration of spaceflight than the longest ISS flights to date. Understanding the pathogenesis; identifying those at increased risk; and mitigating the effects of SANS are of critical importance so that astronauts can complete LDSF missions safely. Terrestrial analogs, that is, experimental models of SANS that can be

\section{REFERENCES}

1. Cappellesso R, Nicole L, Guido A, Pizzol D. Spaceflight osteoporosis: current state and future perspective. Endocr Regul. (2015) 49:231-9. doi: 10.4149/endo_2015_04_231

2. Fitts RH, Trappe SW, Costill DL, Gallagher PM, Creer AC, Colloton PA, et al. Prolonged space flight-induced alterations in the structure and function of human skeletal muscle fibres. J Physiol. (2010) 588(Pt 18):3567-92. doi: 10.1113/jphysiol.2010.188508

3. Lee AG, Mader TH, Gibson CR, Tarver W, Rabiei P, Riascos RF, et al. Spaceflight associated neuro-ocular syndrome (SANS) and the neuro-ophthalmologic effects of microgravity: a review and an update. NPJ Microgravity. (2020) 6:7. doi: 10.1038/s41526-0200097-9 completed on Earth, are an important tool with which to achieve this and HDTBR is a promising one as, with the right parameters, it is associated with many of the ophthalmic findings seen in SANS. Further HDTBR studies may provide useful information by applying advances in ophthalmic and neuro imaging and image analysis.

HDTBR results support genetic traits such as MTRR and SHMT1 as risk factors. HDTBR itself may be a practical way to screen for risk of SANS development in individuals. HDTBR results also support the use of lower body negative pressure as a countermeasure for SANS. Other countermeasures may include artificial gravity, dietary supplementation, varying training regiments, topical or oral medication, non-invasive monitoring, and external device utilization during or before flight/HDTBR. HDTBR may be helpful to identify the best inflight measurements with which to diagnose and monitor SANS.

Beyond government-funded space exploration, private space companies (e.g., SpaceX, Blue Origin) strive to increase the accessibility to spaceflight for civilian populations as space tourism (78-80). Short-term HDTBR may be a practical way to screen civilians for susceptibility to consequences of cephalad shifts. Furthermore, a collaborative effort between private space companies and HDTBR researchers may provide novel understandings to short-term spaceflight in the civilian population when comparing pre/post-flight and HDTBR data. As we head into a new era of spaceflight, HDTBR emerges as a promising terrestrial analog to understand how to optimally protect the neuro-ophthalmic health of civilians and astronauts.

\section{AUTHOR CONTRIBUTIONS}

JO performed the primary literature review and drafted the initial manuscript, tables, and figure, and edited the manuscript. HM and AL provided feedback and edited the manuscript. All authors contributed to the article and approved the submitted version.

\section{FUNDING}

This work was supported by NIH p30 026877, Research to Prevent Blindness unrestricted grant to Stanford Department of Ophthalmology.
4. Stenger MB, Tarver WJ, Brunstetter T, Gibson CR, Laurie SS, Lee S, et al. Evidence Report: Risk of Spaceflight Associated Neuro-Ocular Syndrome (SANS) (Human Research Program Human Health Countermeasures Element) (2017).

5. Saivin S, Pavy-Le Traon A, Cornac A, Guell A, Houin G. Impact of a four-day head-down tilt (-6 degrees) on lidocaine pharmacokinetics used as probe to evaluate hepatic blood flow. J Clin Pharmacol. (1995) 35:697-704. doi: 10.1002/j.1552-4604.1995.tb04110.x

6. Mauran P, Sediame S, Pavy-Le Traon A, Maillet A, Carayon A, Barthelemy C, et al. Renal and hormonal responses to isotonic saline infusion after 3 days' head-down tilt vs. supine and seated positions. Acta Physiol Scand. (2003) 177:167-76. doi: 10.1046/j.1365-201X.2003.01059.x

7. Berney S, Denehy L, Pretto J. Head-down tilt and manual hyperinflation enhance sputum clearance in patients who are intubated and ventilated. 
Aust J Physiother. (2004) 50:9-14. doi: 10.1016/S0004-9514(14) 60243-9

8. Sun XQ, Yao YJ, Yang CB, Jiang CL, Jiang SZ, Liang WB. Effect of lower body negative pressure on orthostatic tolerance and cardiac function during 21 days head-down tilt bed rest. J Gravit Physiol. (2003) 10:11-7.

9. Koppelmans V, Bloomberg JJ, De Dios YE, Wood SJ, Reuter-Lorenz PA, Kofman IS, et al. Brain plasticity and sensorimotor deterioration as a function of 70 days head down tilt bed rest. PLOS ONE. (2017) 12:e0182236. doi: 10.1371/journal.pone.0182236

10. Trudel G, Uhthoff HK, Laneuville O. Hemolysis during and after 21 days of head-down-tilt bed rest. Physiol Rep. (2017) 5:e13469. doi: 10.14814/phy2.13469

11. Koy T, Ganse B, Zange J, Rittweger J, Pohle-Frohlich R, Fings-Meuthen P, et al. T2-relaxation time increases in lumbar intervertebral discs after 21d head-down tilt bed-rest. J Musculoskelet Neuronal Interact. (2017) 17:140-145.

12. Alessandro C, Sarabadani Tafreshi A, Riener R. Cardiovascular responses to leg muscle loading during head-down tilt at rest and after dynamic exercises. Sci Rep. (2019) 9:2804. doi: 10.1038/s41598-019-39360-6

13. Orter S, Mostl S, Bachler M, Hoffmann F, Kaniusas E, Reisinger M, et al. Measuring arterial stiffness in a head-down tilt bed rest study: a multisensor approach(.). Annu Int Conf IEEE Eng Med Biol Soc. (2020) 2020:2715-8. doi: 10.1109/EMBC44109.2020.9176275

14. Koppelmans V, Erdeniz B, De Dios YE, Wood SJ, Reuter-Lorenz PA, Kofman I, et al. Study protocol to examine the effects of spaceflight and a spaceflight analog on neurocognitive performance: extent, longevity, neural bases. BMC Neurol. (2013) 13:205. doi: 10.1186/1471-2377-13-205

15. Chen J, Wall M. Epidemiology and risk factors for idiopathic intracranial hypertension. Int Ophthalmol Clin. (2014) 54:1-11. doi: 10.1097/IIO.0b013e3182aabf11

16. Lee AG, Tarver WJ, Mader TH, Gibson CR, Hart SF, Otto CA. Neuroophthalmology of space flight. J Neuroophthalmol. (2016) 36:85-91. doi: 10.1097/WNO.0000000000000334

17. Mader TH, Gibson CR, Pass AF, Kramer LA, Lee AG, Fogarty J, et al. Optic disc edema, globe flattening, choroidal folds, and hyperopic shifts observed in astronauts after long-duration space flight. Ophthalmology. (2011) 118:2058-69. doi: 10.1016/j.ophtha.2011.06.021

18. Mader TH, Gibson CR, Pass AF, Lee AG, Killer HE, Hansen HC, et al. Optic disc edema in an astronaut after repeat long-duration space flight. $J$ Neuroophthalmol. (2013) 33:249-55. doi: 10.1097/WNO.0b013e31829b41a6

19. Zhang LF, Hargens AR. Spaceflight-induced intracranial hypertension and visual impairment: pathophysiology and countermeasures. Physiol Rev. (2018) 98:59-87. doi: 10.1152/physrev.00017.2016

20. Harris LR, Jenkin M, Jenkin H, Zacher JE, Dyde RT. The effect of longterm exposure to microgravity on the perception of upright. NPJ Microgravity. (2017) 3:3. doi: 10.1038/s41526-016-0005-5

21. Mader TH, Gibson CR, Miller NR, Subramanian PS, Patel NB, Lee AG. An overview of spaceflight-associated neuro-ocular syndrome (SANS). Neurol India. (2019) 67(Suppl.):S206-11. doi: 10.4103/0028-3886.259126

22. Mader TH, Gibson CR, Barratt MR, Miller NR, Subramanian PS, Killer HE. Persistent globe flattening in astronauts following long-duration spaceflight. Neuro Ophthalmol. (2020) 45:29-35. doi: 10.1080/01658107.2020.1791189

23. Nelson ES, Mulugeta L, Myers JG. Microgravity-induced fluid shift and ophthalmic changes. Life. (2014) 4:621-65. doi: 10.3390/life4040621

24. Pandiarajan M, Hargens AR. Ground-based analogs for human spaceflight. Front Physiol. (2020) 11:716. doi: 10.3389/fphys.2020.00716

25. Deng Y, Ridley AJ, Wang W. Effect of the altitudinal variation of the gravitational acceleration on the thermosphere simulation. J Geophys Res Space Phys. (2008) 113. doi: 10.1029/2008JA013081

26. Demontis GC, Germani MM, Caiani EG, Barravecchia I, Passino C, Angeloni D. Human pathophysiological adaptations to the space environment. Front Physiol. (2017) 8:547. doi: 10.3389/fphys.2017.00547

27. Herranz R, Anken R, Boonstra J, Braun M, Christianen PC, de Geest M, et al. Ground-based facilities for simulation of microgravity: organism-specific recommendations for their use, recommended terminology. Astrobiology. (2013) 13:1-17. doi: 10.1089/ast.2012.0876

28. Karmali F, Shelhamer M. The dynamics of parabolic flight: flight characteristics and passenger percepts. Acta Astronaut. (2008) 63:594-602. doi: 10.1016/j.actaastro.2008.04.009
29. Thirsk R, Kuipers A, Mukai C, Williams D. The space-flight environment: the International Space Station and beyond. CMAJ. (2009) 180:1216-20. doi: 10.1503/cmaj.081125

30. Rogers MJ, Vogt GL, Wargo MJ. The Mathematics of Microgravity. Washington, DC: National Aeronautics and Space Administration Educational Brief.

31. Kramer LA, Sargsyan AE, Hasan KM, Polk JD, Hamilton DR. Orbital and intracranial effects of microgravity: findings at 3-T MR imaging. Radiology. (2012) 263:819-27. doi: 10.1148/radiol.12111986

32. Marshall-Goebel K, Stevens B, Rao CV, Suarez JI, Calvillo E, Arbeille P, et al. Internal jugular vein volume during head-down tilt and carbon dioxide exposure in the SPACECOT study. Aerosp Med Hum Perform. (2018) 89:3516. doi: 10.3357/AMHP. 4934.2018

33. Martin DS, Lee SM, Matz TP, Westby CM, Scott JM, Stenger MB, et al. Internal jugular pressure increases during parabolic flight. Physiol Rep. (2016) 4:e13068. doi: 10.14814/phy2.13068

34. Dhoot R, Margolin E. Papilledema, in StatPearls. Treasure Island, FL: StatPearls Publishing (2020).

35. Mader TH, Gibson CR, Otto CA, Sargsyan AE, Miller NR, Subramanian PS, et al. Persistent asymmetric optic disc swelling after long-duration space flight: implications for pathogenesis. J Neuroophthalmol. (2017) 37:133-9. doi: $10.1097 /$ WNO.0000000000000467

36. Thurtell MJ, Bruce BB, Newman NJ, Biousse V. An update on idiopathic intracranial hypertension. Rev Neurol Dis. (2010) 7:e56-68.

37. Killer HE, Jaggi GP, Flammer J, Miller NR, Huber AR, Mironov A. Cerebrospinal fluid dynamics between the intracranial and the subarachnoid space of the optic nerve. Is it always bidirectional? Brain. (2007) $130(\mathrm{Pt}$ 2):514-20. doi: 10.1093/brain/awl324

38. Killer HE, Jaggi GP, Flammer J, Miller NR, Huber AR. The optic nerve: a new window into cerebrospinal fluid composition? Brain. (2006) $129(\mathrm{Pt}$ 4):1027-30. doi: 10.1093/brain/awl045

39. Hao J, Pircher A, Miller NR, Hsieh J, Remonda L, Killer HE. Cerebrospinal fluid and optic nerve sheath compartment syndrome: a common pathophysiological mechanism in five different cases? Clin Exp Ophthalmol. (2020) 48:212-9. doi: 10.1111/ceo.13663

40. Shinojima A, Kakeya I, Tada S. Association of space flight with problems of the brain and eyes. JAMA Ophthalmol. (2018) 136:1075-6. doi: 10.1001/jamaophthalmol.2018.2635

41. Roberts DR, Albrecht MH, Collins HR, Asemani D, Chatterjee AR, Spampinato MV, et al. Effects of spaceflight on astronaut brain structure as indicated on MRI. N Engl J Med. (2017) 377:1746-53. doi: 10.1056/NEJMoa1705129

42. Wahlin A, Holmlund P, Fellows AM, Malm J, Buckey JC, Eklund A. Optic nerve length before and after spaceflight. Ophthalmology. (2021) 128:309-16. doi: 10.1016/j.ophtha.2020.07.007

43. Watenpaugh DE. Analogs of microgravity: head-down tilt and water immersion. J Appl Physiol. (1985) (2016) 120:904-14. doi: 10.1152/japplphysiol.00986.2015

44. Marshall-Goebel K, Ambarki K, Eklund A, Malm J, Mulder E, Gerlach D, et al. Effects of short-term exposure to head-down tilt on cerebral hemodynamics: a prospective evaluation of a spaceflight analog using phase-contrast MRI. J Appl Physiol 1985. (2016) 120:1466-73. doi: 10.1152/japplphysiol.00841. 2015

45. Parazynski SE, Hargens, Alan R, Tucker B, Aratow M, Styf J, et al. Transcapillary fluid shifts in head and neck tissues during and after simulated microgravity. NASA Tech Rep Serv. (1991) 71:2469-75. doi: 10.1152/jappl.1991.71.6.2469

46. Smith JD, Cromwell RL, Kundrot CE, Charles JB. Six-Degree Head-Down tilt Bed Rest: Forty Years of Development as a Physiological Analog for Weightlessness. San Jose, CA: American Society for Gravitational and Space Biology Conference (2011).

47. Thomsen JS, Morukov BV, Vico L, Alexandre C, Saparin PI, Gowin W. Cancellous bone structure of iliac crest biopsies following 370 days of headdown bed rest. Aviat Space Environ Med. (2005) 76:915-22.

48. Laurie SS, Vizzeri G, Taibbi G, Ferguson CR, Hu X, Lee SMC, et al. Effects of short-term mild hypercapnia during head-down tilt on intracranial pressure and ocular structures in healthy human subjects. Physiol Rep. (2017) 5:e13302. doi: 10.14814 /phy2.13302 
49. Taibbi G, Cromwell RL, Zanello SB, Yarbough PO, Ploutz-Snyder RJ, Godley BF, et al. Ocular outcomes comparison between 14- and 70-day head-down-tilt bed rest. Invest Ophthalmol Vis Sci. (2016) 57:495-501. doi: 10.1167/iovs.15-18530

50. Laurie SS, Lee SMC, Macias BR, Patel N, Stern C, Young M, et al. Optic disc edema and choroidal engorgement in astronauts during spaceflight and individuals exposed to bed rest. JAMA Ophthalmol. (2020) 138:165-72. doi: 10.1001/jamaophthalmol.2019.5261

51. Laurie SS, Macias BR, Dunn JT, Young M, Stern C, Lee SMC, et al. Optic disc edema after 30 days of strict head-down tilt bed rest. Ophthalmology. (2019) 126:467-8. doi: 10.1016/j.ophtha.2018.09.042

52. Petersen LG, Lawley JS, Lilja-Cyron A, Petersen JCG, Howden EJ, Sarma S, et al. Lower body negative pressure to safely reduce intracranial pressure. $J$ Physiol. (2019) 597:237-48. doi: 10.1113/JP276557

53. Hughson RL, Yee N, Greaves D. Elevated Inspired and End-Tidal PCO2 on the International Space Station. San Diego, CA: Federation of American Societies for Experimental Biology (2016). p. 762.3.

54. Lee JK, De Dios Y, Kofman I, Mulavara AP, Bloomberg JJ, Seidler RD. Head down tilt bed rest plus elevated $\mathrm{CO} 2$ as a spaceflight analog: effects on cognitive and sensorimotor performance. Front Hum Neurosci. (2019) 13:355. doi: 10.3389/fnhum.2019.00355

55. Laurie SS, Christian K, Kysar J, Lee SMC, Lovering AT, Macias BR, et al. Unchanged cerebrovascular $\mathrm{CO} 2$ reactivity and hypercapnic ventilatory response during strict head-down tilt bed rest in a mild hypercapnic environment. J Physiol. (2020) 598:2491-505. doi: 10.1113/JP279383

56. Roberts DR, Collins HR, Lee JK, Taylor JA, Turner M, Zaharchuk G, et al. Altered cerebral perfusion in response to chronic mild hypercapnia and head-down tilt Bed rest as an analog for Spaceflight. Neuroradiology. (2021). doi: 10.1007/s00234-021-02660-8. [Epub ahead of print].

57. Kergoat H, Lovasik JV. Seven-degree head-down tilt reduces choroidal pulsatile ocular blood flow. Aviat Space Environ Med. (2005) 76:930-4.

58. Pardon LP, Cheng H, Chettry P, Patel NB. Optic nerve head morphological changes over 12 hours in seated and head-down tilt postures. Invest Ophthalmol Vis Sci. (2020) 61:21. doi: 10.1167/iovs.61.13.21

59. Petersen LG, Petersen JC, Andresen M, Secher NH, Juhler M. Postural influence on intracranial and cerebral perfusion pressure in ambulatory neurosurgical patients. Am J Physiol Regul Integr Comp Physiol. (2016) 310:R100-4. doi: 10.1152/ajpregu.00302.2015

60. Xu X, Li L, Cao R, Tao Y, Guo Q, Geng J, et al. Intraocular pressure and ocular perfusion pressure in myopes during $21 \mathrm{~min}$ head-down rest. Aviat Space Environ Med. (2010) 81:418-22. doi: 10.3357/ASEM.2629.2010

61. Zwart SR, Laurie SS, Chen JJ, Macias BR, Lee SMC, Stenger M, et al. Association of genetics and $\mathrm{b}$ vitamin status with the magnitude of optic disc edema during 30-day strict head-down tilt bed rest. JAMA Ophthalmol. (2019). doi: 10.1001/jamaophthalmol.2019.3124. [Epub ahead of print].

62. Charles JB, Lathers CM. Summary of lower body negative pressure experiments during space flight. J Clin Pharmacol. (1994) 34:571-83. doi: 10.1002/j.1552-4604.1994.tb02009.x

63. Marshall-Goebel K, Terlevic R, Gerlach DA, Kuehn S, Mulder E, Rittweger J. Lower body negative pressure reduces optic nerve sheath diameter during head-down tilt. J Appl Physiol 1985. (2017) 123:1139-44. doi: 10.1152/japplphysiol.00256.2017

64. Lawley JS, Babu G, Janssen S, Petersen LG, Hearon CM Jr, Dias KA, et al. Daily generation of a footward fluid shift attenuates ocular changes associated with head-down tilt bedrest. J Appl Physiol 1985. (2020) 129:122031. doi: 10.1152/japplphysiol.00250.2020

65. Arbeille P, Fomina G, Achaibou F, Pottier J, Kotovskaya A. Cardiac and vascular adaptation to $0 \mathrm{~g}$ with and without thigh cuffs (Antares 14 and Altair 21 day Mir spaceflights). Acta Astronaut. (1995) 36:753-62. doi: 10.1016/0094-5765(95)00166-2

66. Macias B, Balasubramanian S, Huang AS, Liu JH, Lee SM, Laurie S. Fluid Shift Induced Alterations of the Optic Nerve Head and Peripapillary Choroid Assessed using Optical Coherence Tomography Investigative Ophthalmology \& Visual Science. Baltimore, MD: ARVO Annual Meeting, Investigative Ophthalmology \& Visual Science (2017).
67. Hackney KJ, Scott JM, Hanson AM, English KL, Downs ME, Ploutz-Snyder LL. The astronaut-athlete: optimizing human performance in space. J Strength Cond Res. (2015) 29:3531-45. doi: 10.1519/JSC.0000000000001191

68. Taibbi G, Cromwell RL, Zanello SB, Yarbough PO, Ploutz-Snyder RJ, Godley $\mathrm{BF}$, et al. Ophthalmological evaluation of integrated resistance and aerobic training during 70-day bed rest. Aerosp Med Hum Perform. (2017) 88:633-40. doi: $10.3357 / A M H P .4768 .2017$

69. Scott JM, Tucker WJ, Martin D, Crowell JB, Goetchius E, Ozgur O, et al. Association of exercise and swimming goggles with modulation of cerebro-ocular hemodynamics and pressures in a model of spaceflightassociated neuro-ocular syndrome. JAMA Ophthalmol. (2019) 137:652-9. doi: 10.1001/jamaophthalmol.2019.0459

70. Nag DS, Sahu S, Swain A, Kant S. Intracranial pressure monitoring: gold standard and recent innovations. World J Clin Cases. (2019) 7:1535-53. doi: 10.12998/wjcc.v7.i13.1535

71. Tymko MM, Boulet LM, Donnelly J. Intracranial pressure in outer space: preparing for the mission to mars. J Physiol. (2017) 595:4587-8. doi: 10.1113/JP274315

72. Kemp D, Ebert D, Danielson R, Marshall-Goebel K, Macias B, Stenger M. Use of otoacousticemission phase change to evaluate countermeasures for spaceflight-associated neuro-ocular syndrome. NASA Tech Rep Serv. (2020). Available online at: https://ntrs.nasa.gov/api/citations/20200001310/ downloads/20200001310.pdf

73. Jerin C, Gurkov R. Posture-induced changes of ocular vestibular evoked myogenic potentials suggest a modulation by intracranial pressure. Exp Brain Res. (2014) 232:2273-9. doi: 10.1007/s00221-014-3 918-9

74. de Carlo TE, Romano A, Waheed NK, Duker JS. A review of optical coherence tomography angiography (OCTA). Int J Retina Vitreous. (2015) 1:5. doi: 10.1186/s40942-015-0005-8

75. Caccavale A, Mignemi L. Fluorescein and indocyanine green angiography findings in a case of poststreptococcal syndrome with erythema nodosum and posterior uveitis. Retina. (2001) 21:669-72. doi: 10.1097/00006982-200112000-00020

76. van Oosterhout WP, Terwindt GM, Vein AA, Ferrari MD. Space headache on earth: head-down-tilted bed rest studies simulating outerspace microgravity. Cephalalgia. (2015) 35:335-43. doi: 10.1177/03331024145 36058

77. Guilliams ME, Nieschwitz B, Hoellen D, Loehr J. Preflight and in-flight exercise conditions for astronauts on the international space station. NASA Tech Rep Serv. (2011). Available online at: https://ntrs.nasa.gov/api/citations/ 20110016265/downloads/20110016265.pdf?attachment=true

78. Stepanek J, Blue RS, Parazynski S. Space medicine in the era of civilian spaceflight. $N$ Engl J Med. (2019) 380:1053-1060. doi: 10.1056/NEJMra1609012

79. Aerospace Medical Association Task Force on Space Travel, Medical guidelines for space passengers. aerospace medical association task force on space travel. Aviat Space Environ Med. (2001) 72:948-50.

80. Witze A. SpaceX to launch astronauts - and a new era of private human spaceflight. Nature. (2020) 582. doi: 10.1038/d41586-020-01554-8

Conflict of Interest: The authors declare that the research was conducted in the absence of any commercial or financial relationships that could be construed as a potential conflict of interest.

The Handling Editor declared a past co-authorship with two of the authors, $\mathrm{AL}$ and HM.

Copyright (C) 2021 Ong, Lee and Moss. This is an open-access article distributed under the terms of the Creative Commons Attribution License (CC BY). The use, distribution or reproduction in other forums is permitted, provided the original author(s) and the copyright owner(s) are credited and that the original publication in this journal is cited, in accordance with accepted academic practice. No use, distribution or reproduction is permitted which does not comply with these terms. 